\title{
Local Conformal Symmetry and its Fate at Quantum Level
}

\section{Ilya Shapiro*i}

Univ. Federal Juiz de Fora

E-mail: shapiro@fisica.ufjf.br

The purpose of this article is to present a short review of local conformal symmetry in curved $4 d$ space-time. Furthermore we discuss the conformal anomaly and anomaly-induced effective actions. Despite the conformal symmetry is always broken at quantum level, it may be a basis of useful and interesting approximations for investigating quantum corrections.

Fifth International Conference on Mathematical Methods in Physics - IC2006

April 24-28 2006

Centro Brasileiro de Pesquisas Fisicas, Rio de Janeiro, Brazil

\footnotetext{
${ }^{*}$ Speaker.

${ }^{\dagger}$ Also at Tomsk State Pedagogical University, Tomsk, Russia.
} 


\section{Introduction}

The local conformal symmetry of matter fields in curved space and proper gravity always attracted a great interest. It is not our aim to list the main publications on the subject nor the main lines of research related to it. So, let us start by mentioning a recent review [1] where one can find some relevant references to start. In our article we shall concentrate on those aspects of the conformal theory in four dimensions, which are relevant for the applications, especially to cosmology. We shall pay special attention to the quantum theory and discuss conformal anomaly and anomaly-induced effective action of gravity. Many other issues will be left aside, some of them may be eventually considered in the extended version of this review article.

In order to understand the reason to introduce a local conformal symmetry, let us start from a very simple example discussed in [2]. Consider a massive scalar field in curved space-time. The minimal action has the form

$$
S=\frac{1}{2} \int \sqrt{-g}\left(g^{\mu \nu} \nabla_{\mu} \varphi \nabla_{v} \varphi+m^{2} \varphi^{2}+\xi R \varphi^{2}\right),
$$

where $\xi=0$. However, the minimal theory is inconsistent at quantum level if we introduce interactions with other fields or scalar self-interaction. In principle, one has to keep the non-minimal parameter $\xi$ arbitrary to provide the multiplicative renormalizability of the theory. At the same time the value $\xi=1 / 6$ is very special, for in the massless case $m=0$ it corresponds to the local conformal symmetry

$$
g_{\mu \nu} \rightarrow g_{\mu \nu}^{\prime}=g_{\mu v} e^{2 \sigma(x)} \quad \varphi \rightarrow \varphi^{\prime}=\varphi e^{-\sigma(x)} .
$$

Now, let us consider the massless limit of the theory from another point of view. Basing on fundamental principles of quantum theory, one is expecting to meet correspondence between the field and particle description of the matter. It is well known that, for the classical particles, the massless limit corresponds to the vanishing trace of the energy-momentum tensor

$$
T_{\mu}^{\mu}=-\frac{2}{\sqrt{-g}} g_{\mu v} \frac{\delta S}{\delta g_{\mu v}}=0 .
$$

However, this identity can be achieved, in the field description (1.1), only for the conformal value $\xi=1 / 6$ of the non-minimal parameter (of course, the relation (1.3) holds only on the mass shell for the scalar field or for the corresponding particles). Therefore, only conformal theory can provide a correct particle-field correspondence in the massless limit. One can see that the conformal value $\xi=1 / 6$ does provide certain advantage at this level. The next question is whether it is possible to maintain the conformal value of $\xi$ and, in general, local conformal symmetry, at the quantum level, when the loop corrections are taken into account. This issue is the main subject of the present review.

The paper is organized as follows. In the next section we shall list known conformal theories in $n=4$ and after that consider the quantum theory, where the local conformal invariance always breaks down. Section 3 is devoted to the brief description of the anomaly. We discuss the main difference between global and local conformal symmetries at quantum level. In section 4 we present, in more details than usual, the derivation of the anomaly-induced effective action of vacuum. In 
section 5 we come back to anomaly and describe its ambiguities in relation to the effective action. Section 6 contains a brief description of the situation in conformal quantum gravity. Finally, in section 7 we draw our conclusions.

\section{Particular examples of conformal theories}

Consider a general metric-scalar theory [3]

$$
S=\int d^{4} x \sqrt{-g}\left\{A(\phi)(\nabla \phi)^{2}+B(\phi) R+C(\phi)\right\}
$$

and perform the local conformal transformation of $g_{\mu \nu}$ plus an arbitrary scalar reparametrizations (thus, generalizing the eq. (1.2)). In order to make things simpler, we start from the action without kinetic term for the scalar field [4] and transform it to (2.1)

$$
S=\int d^{4} x \sqrt{g^{\prime}}\left\{R^{\prime} \Phi+V(\Phi)\right\}, \quad g_{\mu \nu}^{\prime}=g_{\mu \nu} e^{2 \sigma(\phi)}, \quad \Phi=\Phi(\phi) .
$$

Simple calculation leads to the relation

$$
A(\phi)=6 e^{2 \sigma(\phi)}\left[\Phi \sigma_{1}+\Phi_{1}\right] \sigma_{1}, \quad B(\phi)=\Phi(\phi) e^{2 \sigma}
$$

where $B_{1}=d B / d \phi$ etc. One can see that the absence of the kinetic term in the action (2.2) does not mean that this field is not dynamical. The dynamics of the scalar field is due to the interaction with the scalar mode of the metric. For instance, the free minimal scalar field plus General Relativity (GR) is the particular case of the action (2.1) and is conformally equivalent to (2.2).

The conformal symmetry of the action corresponds to pure GR, $\Phi=$ const. Then

$$
A=\frac{3 B_{1}}{2 B^{2}}, \quad C=\lambda B^{2}, \quad \text { where } \quad B=B(\phi), C=C(\phi), B_{1}=\frac{d B}{d \phi} .
$$

The well-known particular case of the theory satisfying the constraints (2.4) is (1.1) with $m=0$, $\xi=1 / 6$ and with an additional self-interaction term. One can rewrite it in the form

$$
S=\frac{1}{2} \int d^{4} x \sqrt{-g}\left(-\phi \Delta_{2} \phi+\frac{\lambda}{12} \phi^{4}\right), \quad \text { where } \quad \Delta_{2}=\square-R / 6 .
$$

All models which satisfy (2.4) are linked by conformal transformation of the metric plus scalar reparametrization [3].

Other conventional examples of conformal fields include massless spinor and vector

$$
\begin{array}{r}
S_{1 / 2}=\frac{i}{2} \int d^{4} x \sqrt{g}\left\{\bar{\psi} \gamma^{\mu} \nabla_{\mu} \psi-\nabla_{\mu} \bar{\psi} \gamma^{\mu} \psi\right\} \\
S_{1}=-\frac{1}{4} \int d^{4} x \sqrt{g} F_{\mu v} F^{\mu \nu}
\end{array}
$$

with the transformation rules

$$
g_{\mu \nu} \rightarrow g_{\mu \nu}^{\prime}=g_{\mu \nu} e^{2 \sigma}, \quad A_{\mu} \rightarrow A_{\mu}^{\prime}=A_{\mu}, \quad \psi \rightarrow \psi^{\prime}=\psi e^{-3 \sigma / 2}, \quad \bar{\psi} \rightarrow \bar{\psi}^{\prime}=\bar{\psi} e^{-3 \sigma / 2}
$$


Let us notice that the difference between conformal weight and dimension for the vector field is due to the vector field definition in curved space-time ${ }^{1}$

$$
A_{\mu}=A_{b} e_{\mu}^{b}, \quad e_{\mu}^{b} e_{v}^{a} \eta_{a b}=g_{\mu v}, \quad e_{\mu}^{b} e_{v}^{a} g^{\mu v}=\eta^{a b} .
$$

The importance of this observation is that it shows a direct relation between local and global conformal symmetries. The generalization to the non-Abelian case is straightforward.

The interactions between usual vectors, scalars and fermions are always conformal if the corresponding coupling constants have zero mass dimension. Hence gauge, Yukawa and $\phi^{4}$ interaction terms are conformal while $\phi^{3}$ is not.

The last conventional example is the conformal (Weyl) gravity, which includes only metric field

$$
S_{W}=\int d^{4} x \sqrt{g} C^{2}(4), \quad C^{2}(n)=R_{\mu v \alpha \beta}^{2}-\frac{4}{n-2} R_{\mu \nu}^{2}+\frac{1}{(n-1)(n-2)} R^{2},
$$

where $n$ is the space-time dimension. The main difference between the Weyl gravity model (2.10) and GR is that the former does not produce the correct Newton limit. This is of course natural because the coupling constant in this theory is dimensionless and therefore an additional mechanism is needed in order to produce a dimensional parameter such as Newton constant. The most natural option is to consider the Weyl gravity and the conformal scalar field together. In this case the quantum effects lead to the complicated effective potential for the scalar field. This may produce a dimensional transmutation and eventually lead to induced GR with induced values of both Newton and cosmological constants. A general review on the induced gravity approach can be found in [5]. There are several possible mechanism of how this method can be applied to the initially conformal theory $[6,7,8]$. We will not discuss this aspect of the conformal theory in what follows, because this review is of a short kind. Instead, we shall concentrate on a more basic phenomenon (conformal anomaly) in the next section.

The main difference between the conformal scalar, fermion and vector cases presented above and the last example of Weyl gravity is that it is a fourth derivative theory while the matter fields cases are all described by lower derivative theories. However one can construct also examples of conformal higher derivative scalars and fermions (and perhaps vectors, despite this has not been done yet) which possess the local conformal invariance.

Let us start with scalars and consider two alternative different models. The fourth derivative scalar of the first kind has an action $[9,10]$

$$
\begin{aligned}
S_{4} & =\int d^{4} x \sqrt{g} \varphi \Delta_{4} \varphi, \\
\text { where } \quad \Delta_{4} & =\square^{2}+2 R^{\mu v} \nabla_{\mu} \nabla_{v}-\frac{2}{3} R \square+\frac{1}{3} R_{; \mu} \nabla^{\mu} .
\end{aligned}
$$

The conformal transformation law for this scalar is $\varphi \rightarrow \varphi^{\prime}$. The importance of the model (2.11) is based on its use for integrating conformal anomaly. We shall discuss this point in the next section.

The fourth derivative scalar of the second kind can be presented, up to reparametrization of scalar $\chi=\chi(\phi)$, in the form [11]

$$
\int d^{4} x \sqrt{-g}\left\{\left(\phi^{-1} \Delta_{2} \phi\right)^{2}-a \phi \Delta_{2} \phi-b \phi^{4}+c C^{2}+d E\right\},
$$

\footnotetext{
${ }^{1}$ I am grateful to Joan Solà, who called my attention to this point.
} 
where $a, b, c, d$ are some constants. This model is a direct higher derivative generalization of the usual conformal scalar theory (2.5) and the transformation laws for metric and scalar are of course identical. The complete form of the parametrization-invariant higher derivative action, similar to (2.1) with the constraints (2.3) satisfied, has been constructed in [12]. One can notice that the above two theories represent very particular cases of the general fourth derivative metric-scalar model formulated in [13]. This general model involves 12 arbitrary functions of the scalar (in fact 11 , because one may be always included into scalar parametrization), while both models presented above have no such functions.

Let us remark that both fourth derivative scalar models (2.11) and (2.13) can be generalized to an arbitrary dimension $n \neq 2$. For the case of (2.11) this task has been completed in [12] and for the case of (2.13) the procedure is obvious due to the known prescription for the usual conformal scalar (2.5).

The next example is a third derivative spinor field. In this case, again, the conformal invariance is provided by introducing the higher derivatives, changing the transformation law for the field and adjusting the parameters of the higher order differential operator $[14,15]$. The action of the model is

$$
S_{3}=\frac{i}{2} \int d^{4} x \sqrt{-g}\left\{\bar{\psi} \gamma^{\mu} \mathscr{D}_{\mu} \psi-\mathscr{D}_{\mu} \bar{\psi} \gamma^{\mu} \psi\right\}
$$

where the self-adjoint third order operator has the form

$$
\mathscr{D}_{\mu}=\nabla_{\mu} \square+R_{\mu \nu} \nabla^{v}-\frac{5}{12} R \nabla_{\mu}-\frac{1}{12}\left(\nabla_{\mu} R\right) .
$$

The transformation law for the spinor $\psi$ is

$$
\psi \rightarrow \psi^{\prime}=\psi e^{-\sigma / 2}, \quad \bar{\psi} \rightarrow \bar{\psi}^{\prime}=\bar{\psi} e^{-\sigma / 2}, \quad \sigma=\sigma(x) .
$$

The natural question is whether is it possible to construct more examples of conformal fields? Obviously, those can be vectors, scalars or spinors with greater number of derivatives (see, e.g. [16, 17] for the works in this direction.). Furthermore it can be spin-3/2 field with higher derivatives, etc. In all cases the construction of symmetric actions can be performed in a way described in [15] for the theory (2.14).

\section{Conformal anomaly in the semiclassical theory}

In this section we shall consider the anomalous violation of the local conformal symmetry in the case of quantum matter on classical curved background. This theory is also known as semiclassical gravity, because it shares many features with quantum theory of gravity. The semiclassical approach is very important independent whether we consider it or not as an approximation to quantum gravity. The reason is that the quantum fields on curved background definitely exist in nature while the reality of quantum gravity is under question. It might occur, after all, that the gravity should not be quantized and, instead, it is an interaction induced by, e.g. (super)string theory in the low-energy limit.

The first step is to consistently formulate the action on classical curved background. The standard criteria for the action of external metric field are (see, e.g. [18]) a) locality of the vacuum 
action, b) renormalizability and c) what one can call simplicity, e.g. we assume there are no $\left[m^{-1}\right]$ parameters or, in other words, we include the minimal set of terms which satisfy $a$ ) and $b$ ) conditions.

The action of vacuum which satisfies these necessary conditions has the form

$$
S_{v a c}=S_{E H}+S_{H D}
$$

where $S_{E H}$ is the Einstein-Hilbert action with cosmological term and

$$
S_{H D}=\int d^{4} x \sqrt{-g}\left\{a_{1} C^{2}+a_{2} E+a_{3} \square R+a_{4} R^{2}\right\} .
$$

Here and below we use the following notations

$$
E=R_{\mu \nu \alpha \beta}^{2}-4 R_{\alpha \beta}^{2}+R^{2}
$$

is the Gauss-Bonnet term (Euler density in $n=4$ ). We avoid using the letter $G$ to denote this quantity because it may be confused with the Newton constant.

In the case of conformal theory at the one-loop level it is sufficient to consider the simplified vacuum action

$$
S_{\text {conf. vac }}=\int d^{4} x \sqrt{-g}\left\{a_{1} C^{2}+a_{2} E+a_{3} \square R\right\} .
$$

Let us emphasize that it is not impossible to add the Einstein-Hilbert action, cosmological constant or the $\int \sqrt{-g} R^{2}$ term here. The statement is that these terms are not really necessary at the one-loop level. In fact, beyond the one-loop approximation the $\int \sqrt{-g} R^{2}$ terms becomes also necessary, this means the conformal theory is not consistent beyond one loop. In case of broken symmetry and generated masses of the matter fields (e.g. through the Coleman-Weinberg mechanism), other mentioned terms may also become necessary.

Now we are in a position to consider the conformal anomaly. We assume the theory includes the metric $g_{\mu \nu}$ as a background field and also quantized matter fields $\Phi$. We denote, furthermore, $k_{\Phi}$ the conformal weight of the field.

The Noether identity for the local conformal symmetry

$$
\left[-2 g_{\mu \nu} \frac{\delta}{\delta g_{\mu \nu}}+k_{\Phi} \Phi \frac{\delta}{\delta \Phi}\right] S\left(g_{\mu \nu}, \Phi\right)=0
$$

produces $T_{\mu}^{\mu}=0$ on shell (1.3).

At quantum level $S_{v a c}\left(g_{\mu v}\right)$ has to be replaced by the effective action of vacuum $\Gamma_{v a c}\left(g_{\mu \nu}\right)$. For the free fields only 1-loop order is relevant and (see [18] for the introduction and further references)

$$
\Gamma_{d i v}=\frac{1}{\varepsilon} \int d^{4} x \sqrt{g}\left\{\beta_{1} C^{2}+\beta_{2} E+\beta_{3} \square R\right\}
$$

where $\varepsilon$ is the regularization parameter. For instance, it is $\varepsilon=\mu^{n-4} /(n-4)$ in dimensional regularization. In the case of global conformal symmetry, the renormalization group method or $\zeta$-regularization tell us $[19,20,18]$

$$
<T_{\mu}^{\mu}>=\left\{\beta_{1} C^{2}+\beta_{2} E+a^{\prime} \square R\right\}
$$


where $a^{\prime}=\beta_{3}$. However, in the case of local conformal invariance there is an ambiguity in the parameter $a^{\prime}[21,22,23]$.

One can derive the anomaly in many different ways, which mainly differ by the regularization choice [24, 25] (see, e.g. [21] for the list of results in some regularizations). Recently, we have analyzed the source of the ambiguity in full details $[23,26]$ and, in particular, have shown that the ambiguity is always related to the local terms in the anomaly-induced effective action of vacuum (see the next section) which have different form from the terms in the classical (conformal invariant) action. It turns out that the dimensional regularization does not enable one to control these local terms and therefore the corresponding terms in the anomaly (which are always total derivatives) remain arbitrary. On the other hand, in other regularizations such as point-splitting one, there is no apparent freedom and it seems that the ambiguity is not there. The same is true if one derives the local term in the anomaly via the heat-kernel solution for the effective action ${ }^{2}$ [28] or makes a massless limit in the effective action of massive fields [23]. Finally, in the covariant Pauli-Villars regularization one can observe the same ambiguity (in a somehow reduced form) and thus confirm the validity of the situation discovered in the dimensional regularization approach. We consider the mentioned ambiguity in some details in section 5 .

Let us consider, as an example, the derivation of anomaly through the most explicit method of dimensional regularization [24]. The theory of matter includes the following set of massless fields: $N_{0}$ scalars (spin-0), $N_{1 / 2}$ spinors (Dirac, spin-1/2) and $N_{1}$ Abelian vectors (spin-1). All $N$ 's indicate a number of fields (not multiplets) in curved space-time, taking conformal version for scalars. We are interested in the vacuum effects and therefore, at one-loop order, can restrict consideration by the free fields case. Using the well-known results (see, e.g. [21, 18]) we arrive at the expression for divergences (3.6) with

$$
\begin{aligned}
& \beta_{1}=-\frac{1}{(4 \pi)^{2}}\left(\frac{N_{0}}{120}+\frac{N_{1 / 2}}{20}+\frac{N_{1}}{10}\right), \\
& \beta_{2}=\frac{1}{(4 \pi)^{2}}\left(\frac{N_{0}}{360}+\frac{11 N_{1 / 2}}{360}+\frac{31 N_{1}}{180}\right), \\
& \beta_{3}=-\frac{1}{(4 \pi)^{2}}\left(\frac{N_{0}}{180}+\frac{N_{1 / 2}}{30}-\frac{N_{1}}{10}\right) .
\end{aligned}
$$

The renormalized one-loop effective action has the form

$$
\Gamma_{R}=S+\bar{\Gamma}+\Delta S
$$

where $\bar{\Gamma}=\bar{\Gamma}_{d i v}+\bar{\Gamma}_{f i n}$ is the naive quantum correction to the classical action and $\Delta S$ is a counterterm. The classical action is $S=S_{\text {matter }}+S_{v a c}$, where $S_{v a c}$ has the form (3.1). Indeed, only conformal invariant part of the vacuum action must be used in (3.9).

$\Delta S$ in (3.9) is an infinite local counterterm which is called to cancel the divergent part of $\bar{\Gamma}$ (3.8). Indeed $\Delta S$ is the only source of the noninvariance of the effective action, since naive (but divergent) contributions of quantum matter fields are conformal. The anomalous trace is therefore

\footnotetext{
${ }^{2}$ Let us notice that the solution for the anomaly-induced effective action presented in the next section agrees with the one obtained from the heat-lernel method, despite this is not easy to see [27].
} 
equal to

$$
T=<T_{\mu}^{\mu}>=-\left.\frac{2}{\sqrt{-g}} g_{\mu \nu} \frac{\delta \Gamma_{R}}{\delta g_{\mu \nu}}\right|_{D=4}=-\left.\frac{2}{\sqrt{-g}} g_{\mu \nu} \frac{\delta \Delta S}{\delta g_{\mu \nu}}\right|_{D=4} .
$$

The calculation of this expression can be done, in a most simple way, as follows. Let us change the parametrization of the metric to

$$
g_{\mu v}=\bar{g}_{\mu v} \cdot e^{2 \sigma}
$$

where $\bar{g}_{\mu v}$ is the fiducial metric with fixed determinant. There is a useful relation

$$
-\frac{2}{\sqrt{-g}} g_{\mu \nu} \frac{\delta A\left[g_{\mu \nu}\right]}{\delta g_{\mu \nu}}=-\left.\frac{1}{\sqrt{-\bar{g}}} e^{-4 \sigma} \frac{\delta A\left[\bar{g}_{\mu \nu} e^{2 \sigma}\right]}{\delta \sigma}\right|_{\bar{g}_{\mu \nu} \rightarrow g_{\mu \nu}, \sigma \rightarrow 0} .
$$

At that point we need a transformation laws for the structures presented in (3.6). They can be found, for instance, in [29], so we will not reproduce these formulas here. It is sufficient to show a single relation between the expression depending on the original metric $g_{\mu \nu}$ and the transformed one

$$
g_{\mu v}^{\prime}=g_{\mu \nu} e^{2 \sigma(x)}, \quad \int \sqrt{-g^{\prime}} C^{2}(n)=\int \sqrt{-g^{\prime}} e^{(n-4) \sigma} C^{2}(n) .
$$

All other expressions of our interest have the same factor $e^{(n-4) \sigma}$ and, on the top of that, some extra terms with derivatives of $\sigma(x)$. For all terms which are not total derivatives, these terms are irrelevant due to the limit procedure in eq. (3.12).

In the simplest case of global conformal factor $\sigma=\lambda=$ const we immediately arrive at the expression (3.7) with $a^{\prime}=\beta_{3}$. However in the local case $\sigma=\sigma(x)$ the situation is more complicated. It is worth mentioning that the left hand side in (3.12) gives zero when applied to the integral of the total derivative term $\int \sqrt{-g} \square R$. We shall come back to discuss this term and the corresponding ambiguity in section 5 .

\section{Anomaly-induced action of vacuum}

One can use conformal anomaly to construct the equation for the finite part of the 1-loop correction to the effective action (we change notations here for the sake of convenience)

$$
\frac{2}{\sqrt{-g}} g_{\mu v} \frac{\delta \bar{\Gamma}_{i n d}}{\delta g_{\mu v}}=\frac{1}{(4 \pi)^{2}}\left(a C^{2}+b E+c \square R\right) .
$$

The solution of this equation is straightforward [10] (see also generalizations for the theory with torsion [30] and with a scalar field [31]). The simplest possibility is to parametrize metric as in (3.13), separating the conformal factor $\sigma(x)$ and rewrite the eq. (4.1) using (3.12). The solution for the effective action is

$$
\begin{gathered}
\bar{\Gamma}=S_{c}\left[\bar{g}_{\mu \nu}\right]+\frac{1}{(4 \pi)^{2}} \int d^{4} x \sqrt{-\bar{g}}\left\{a \sigma \bar{C}^{2}+b \sigma\left(\bar{E}-\frac{2}{3} \bar{\square} \bar{R}\right)+2 b \sigma \bar{\Delta}_{4} \sigma-\right. \\
\left.\left.-\frac{1}{12}\left(c+\frac{2}{3} b\right)\left[\bar{R}-6(\bar{\nabla} \sigma)^{2}-(\bar{\square} \sigma)\right]^{2}\right)\right\}
\end{gathered}
$$


where $S_{c}\left[\bar{g}_{\mu v}\right]=S_{c}\left[g_{\mu \nu}\right]$ is an unknown functional of the metric, which serves as an integration constant for the eq. (4.1).

The solution (4.2) has the merit of being simple, but an important disadvantage is that it is not covariant or, in other words, it is not expressed in terms of original metric $g_{\mu \nu}$. In order to obtain the non-local covariant solution and after represent it in the local form using auxiliary fields, we shall follow [10, 32].

First one has to establish the following relations [10] (see also [29] for details):

$$
\begin{gathered}
\sqrt{-g} C^{2}=\sqrt{-\bar{g}} \bar{C}^{2}, \quad \sqrt{-\bar{g}} \bar{\Delta}_{4}=\sqrt{-g} \Delta_{4}, \\
\sqrt{-g}\left(E-\frac{2}{3} \square R\right)=\sqrt{-\bar{g}}\left(\bar{E}-\frac{2}{3} \square \bar{R}+4 \bar{\Delta}_{4} \sigma\right)
\end{gathered}
$$

and also introduce the Green function for the operator (2.12) $\quad \Delta_{4, x} G(x, y)=\delta(x, y)$. Using these formulas and (3.12) we find, for any $A=A\left(\bar{g}_{\mu \nu}, \sigma\right)$, the relation

$$
\left.\frac{\delta}{\delta \sigma(y)} \int d^{4} x \sqrt{-g(x)} A\left(E-\frac{2}{3} \square R\right)\right|_{g_{\mu \nu}=\bar{g}_{\mu \nu}}=4 \sqrt{-\bar{g}} \bar{\Delta}_{4} A=4 \sqrt{-g} \Delta_{4} A .
$$

In particular, we obtain

$$
\begin{gathered}
\left.\frac{\delta}{\delta \sigma(y)} \int d^{4} x \sqrt{-g(x)} \int d^{4} y \sqrt{-g(y)} a C^{2}(x) G(x, y) \frac{1}{4}\left(E-\frac{2}{3} \square R\right)_{y}\right|_{g_{\mu \nu}=\bar{g}_{\mu \nu}}= \\
=\int d^{4} x \sqrt{-\bar{g}(x)} \bar{\Delta}_{4}(x) \bar{G}(x, y) a \bar{C}^{2}(x)=a \sqrt{-g} C^{2}(y) .
\end{gathered}
$$

Hence the term in the effective action, which produces $T_{a}=-a C^{2}$, is

$$
\Gamma_{a}=\frac{a}{4} \int d^{4} x \sqrt{-g(x)} \int d^{4} y \sqrt{-g(y)} C^{2}(x) G(x, y)\left(E-\frac{2}{3} \square R\right)_{y}
$$

Similarly one can check that the variation (3.12) produces $T_{b}=b\left(E-\frac{2}{3} \square R\right)$ if

$$
\Gamma_{b}=\frac{b}{8} \int d^{4} x \sqrt{-g(x)} \int d^{4} y \sqrt{-g(y)}\left(E-\frac{2}{3} \square R\right)_{x} G(x, y)\left(E-\frac{2}{3} \square R\right)_{y}
$$

Finally, the third constituent of the induced action is the local expression

$$
\Gamma_{c}=-\frac{c+\frac{2}{3} b}{12(4 \pi)^{2}} \int d^{4} x \sqrt{-g(x)} R^{2}(x)
$$

The covariant solution of eq. (4.1) is a sum of (4.7),(4.8) and (4.9).

Our next task is to rewrite the nonlocal expressions obtained above using some auxiliary scalar fields. Let us notice that there are two distinct ways of doing that, leading to the slightly different results. The first option is to introduce the auxiliary fields as a quantum objects, such that, after Gaussian integration over them, we should come back to the non-local expression described above. Another possibility ${ }^{3}$ is to consider auxiliary fields as purely classical objects. After using the

\footnotetext{
${ }^{3}$ Author is grateful to Roberto Balbinot for discussion concerning this point.
} 
classical equations of motion for these fields and replacing them back to the action we come to the original non-local expressions. The difference between the two approaches is that, in the first case, one has to account for the contributions of the auxiliary fields to the anomaly. As a result, the coefficients $a, b, c$ in (4.1) get modified [10]. In this article we will not account for this modification and follow the second approach.

As a first step the remaining terms can be rewritten in the symmetric form

$$
\begin{aligned}
\Gamma_{a, b} & =\int d^{4} x \sqrt{-g(x)} \int d^{4} y \sqrt{-g(y)}\left(E-\frac{2}{3} \square R\right)_{x} G(x, y)\left[\frac{a}{4} C^{2}-\frac{b}{8}\left(E-\frac{2}{3} \square R\right)\right]_{y} \\
& =-\frac{b}{8} \int d^{4} x d^{4} y \sqrt{g(y) g(x)}\left[\left(E-\frac{2}{3} \square R\right)-\frac{a}{b} C^{2}\right]_{x} G(x, y)\left[\left(E-\frac{2}{3} \square R\right)-\frac{a}{b} C^{2}\right]_{y} \\
& +\frac{1}{2} \int d^{4} x d^{4} y \sqrt{g(y) g(x)}\left(\frac{a}{2 \sqrt{b}} C^{2}\right)_{x} G(x, y)\left(\frac{a}{2 \sqrt{b}} C^{2}\right)_{y}
\end{aligned}
$$

The last two terms are appropriate objects for rewriting them using the auxiliary fields. In this way we arrive at the following final expression for the anomaly generated effective action of gravity.

$$
\begin{aligned}
\Gamma & =S_{c}\left[g_{\mu v}\right]-\frac{3 c+2 b}{36(4 \pi)^{2}} \int d^{4} x \sqrt{-g(x)} R^{2}(x)+\int d^{4} x \sqrt{-g(x)}\left\{\frac{1}{2} \varphi \Delta_{4} \varphi-\frac{1}{2} \psi \Delta_{4} \psi\right. \\
& \left.+\varphi\left[\frac{\sqrt{b}}{8 \pi}\left(E-\frac{2}{3} \square R\right)-\frac{a}{8 \pi \sqrt{b}} C^{2}\right]+\frac{a}{8 \pi \sqrt{b}} \psi C^{2}\right\} .
\end{aligned}
$$

Some remarks are in order.

1) The local covariant form (4.11) is dynamically equivalent to the non-local covariant form. The complete definition of the Cauchy problem in the theory with the non-local action requires defining the boundary conditions for the Green functions $G(x, y)$, which shows up independently in the two terms (4.7) and (4.8). The same can be achieved, in the local version, by imposing the boundary conditions on the two auxiliary fields $\varphi$ and $\psi$.

2) The kinetic term for the auxiliary field $\varphi$ is positive while for $\sigma$ it was negative. For $\psi$ the kinetic term has negative sign. The wrong sign does not lead to problems here, because both fields are auxiliary and do not propagate independently.

3) We introduced the new structure $\int C_{x}^{2} G(x, y) C_{y}^{2}$ into the action, despite it was not directly produced by anomaly. This term is indeed conformal invariant and therefore its emergence may be viewed as a simple redefinition of the conformal invariant functional $S_{c}\left[g_{\mu \nu}\right]$. On the other hand, writing the non-conformal terms in the symmetric form (4.10), we have modified the four point function in a very essential way. Therefore, introducing the mentioned conformal term we have just restored the basic structure of the terms generated by anomaly. For this reason, the emergence of the second auxiliary scalar [32] is not an artificial procedure but represents a necessary element of writing the induced action in a local form ${ }^{4}$.

4) The second scalar also proved useful for applications. In particular, the vacuum states of the black hole (Boulware, Hartle-Hawking and Unruh) can be classified through the choice of initial conditions for the two auxiliary fields [34]. Let us stress that this can not be accomplished by

\footnotetext{
${ }^{4}$ The effective action (reffinaction) has been introduced in the paper [32]. Qualitatively similar manner of introducing second scalar has been suggested later on in [33].
} 
using only one field $\varphi$. Therefore the correspondence with other approaches to Hawking radiation indicates that our considerations about the correctness of introducing the second auxiliary scalar are correct.

5) Another important application of the anomaly-induced action is the model of anomalyinduced inflation [2, 31], or Modified Starobinsky Model. In this case the behaviour of conformal factor of the metric is not affected by the presence of the second auxiliary scalar. However, for investigating the evolution of gravitational waves specifying the initial data for both scalars is essential and the situation is close to the one in the black hole case.

\section{Ambiguity of local/surface anomalous terms}

The ambiguity of the local anomalous term $\int \sqrt{-g} R^{2}$ in the effective action and the corresponding term $\square R$ in the anomaly can be observed either in dimensional or in covariant PauliVillars regularization [23].

Let us start from the dimensional regularization and come back to the relation (3.10). As we have already mentioned in section 3, the counterterm $\int \sqrt{-g} \square R$ does not contribute to the anomaly of local conformal symmetry. Hence the anomaly comes from the $\int \sqrt{-g} C^{2}(d)$-type counterterm. However, the requirements of finiteness of renormalized effective and the locality leave us the freedom in choosing the parameter $d$. If we take $d=n+\gamma \cdot[n-4]$, where $\gamma$ is an arbitrary parameter, we meet $a^{\prime} \sim \gamma$ and therefore the coefficient $a$ is arbitrary. It is easy to see that this arbitrariness is equivalent to adding $\int R^{2}$-term to the classical action.

The same result can be achieved in the covariant Pauli-Villars regularization, where one has to introduce a set of massive "regulator" fields. For example, in the case of a massless conformal scalar $\varphi$ we have to start from the action

$$
S_{\text {reg }}=\sum_{i=0}^{N} \int d^{4} x \sqrt{g}\left\{\left(\nabla \varphi_{i}\right)^{2}+\left(\xi_{i} R+m_{i}^{2}\right) \varphi_{i}^{2}\right\} .
$$

The physical scalar field $\varphi \equiv \varphi_{0}$ is conformal $\xi=1 / 6, m_{0}=0$ and bosonic $s_{0}=1$, while PV regulators $\varphi_{i}$ are massive $m_{i}=\mu_{i} M$ and can be bosonic $s_{i}=1$ or fermionic $s_{i}=-2$.

The UV limit $M \rightarrow \infty$ produces the vacuum Eff. Action. The calculation is based on our result for the EA of the massive scalar. We assume that the Pauli-Villars regulators may have conformal $\xi_{i}=1 / 6$ or non-conformal couplings $\xi_{i} \neq 1 / 6$.

The regularized effective action is

$$
\bar{\Gamma}_{\text {reg }}^{(1)}=\lim _{\Lambda \rightarrow \infty} \sum_{i=0}^{N} s_{i} \bar{\Gamma}_{\mathrm{i}}^{(1)}\left(m_{i}, \xi_{i}, \Lambda\right),
$$

where $\Lambda$ is an auxiliary momentum cut-off. It is important that we possess the explicit expression of the $\mathscr{O}\left(R^{2}\right)$ effective action of massive fields derived in [35]. The conformal anomaly is [23]

$$
T=\frac{1}{(4 \pi)^{2}}\left[\frac{1}{180} E-\frac{1}{120} C^{2}+\left(12 \delta-\frac{1}{180}\right) \square R\right], \quad \delta=\sum_{i=1}^{N} s_{i}\left(\xi_{i}-\frac{1}{6}\right)^{2} \ln \mu_{i}^{2},
$$

Exactly as in the dimensional regularization case, the ambiguity is equivalent to the freedom of adding the finite $\int \sqrt{-g} R^{2}$ term and can be fixed only by imposing the renormalization condition 
for this term. The qualitative result is that the definition of the local finite part of the quantum corrections is ambiguous, even if the corresponding term is not present in the classical action and is not renormalized. In order to fix the ambiguity one has to use renormalization condition and for that it is necessary to introduce the non-conformal local term into the classical action. As a result the theory is not conformal anymore. This consideration can be generalized for the case of more general (non purely metric) backgrounds [26], where the situation is similar albeit somehow more complicated.

\section{Weyl quantum gravity}

Finally, let us consider the problem of anomalous violation of local conformal symmetry in the conformal Weyl quantum gravity. The action of the theory has the form

$$
S_{W}=\int d^{4} x \sqrt{g}\left\{\frac{1}{2 \lambda} C^{2}-\frac{1}{\rho} E+\tau \square R\right\},
$$

The action (6.1) is conformal invariant in a sense it satisfies the conformal Noether identity

$$
-\frac{2}{\sqrt{-g}} g_{\mu v} \frac{\delta S_{W}}{\delta g_{\mu v}}=0
$$

The conformal theory (6.1) may be an interesting model of quantum gravity [36, 37, 18], while General Relativity may be induced at quantum level if the Weyl gravity is coupled to a conformal scalar $[6,7,8]$.

The theory (6.1) is the particular case of the general higher derivative quantum gravity which is know to be renormalizable $[38,39]$. At the same time the properties of conformal theory may be quite different from the general one. One can formulate two main questions concerning the properties of the conformal theory at quantum level.

1) Whether the one-loop and/or higher loop infinite renormalization of this theory is conformal invariant. In other words, whether the conformal theory is multiplicatively renormalizable.

2) Whether the anomalous violation of local conformal symmetry occurs in the finite part of the effective action. If so, we need to know whether the corresponding ambiguities, similar to the ones discussed in the previous section, take place here.

The derivation of the one-loop divergences in the conformal theory has been performed in [36, 40, 41]. The result obtained in [41] with the method including rigid automatic control of the calculations fits with the previous ones and has the form

$$
\Gamma_{\mathrm{div}}^{(1)}=-\frac{1}{\varepsilon} \int d^{4} x \sqrt{-g}\left\{\frac{87}{20} E-\frac{199}{30} C^{2}\right\} .
$$

In $n=4$, the dependence on the Gauss-Bonnet term is absent, as it was anticipated earlier $[36,40]$. At the same time this dependence is essential for the renormalization group near four dimensions, producing a number of new nontrivial fixed points, some of them UV stable and some IR stable. There is no real need to calculate the remaining $\int \square R$-type counterterm, because it is gauge fixing dependent [36, 32]. According to (6.3), there is no need for the $\int d^{4} x \sqrt{-g} R^{2}$-type counterterm and, correspondingly, no need to use the so-called special conformal regularization $[42,36]$. At one loop the theory is multiplicatively renormalizable in the usual sense. 
The derivation of anomaly may proceed in exactly the same way as in the semiclassical theory. In particular, the anomaly associated to the divergences (6.3) is well defined and the corresponding non-local terms in the induced action can be obtained in a unique way. At the same time, the local $\int \sqrt{-g} R^{2}$-term is plagued by double ambiguity: due to the gauge dependence of the corresponding $\square R$-type divergence and because of the renormalization-scheme dependence. Finally, the only way to arrive at the well defined quantum corrections is to violate the conformal symmetry at the classical level already. In case this violation is weak, the quantum corrections will respect the corresponding hierarchy.

\section{Conclusions}

We have reviewed some important aspects of local conformal symmetry and in particular its violation at the quantum level by anomaly. There is a variety of theories with local conformal symmetry in $n=4$. Along with the conventional scalar, spinor and vector cases, there are different higher derivative conformal models with higher derivatives.

In the semiclassical theory local conformal symmetry is violated by the trace anomaly. There are both local and non-local terms in the effective action behind the anomaly, but the local terms are plagued by ambiguities, indicating certain inconsistency in the quantum corrections. For conformal quantum gravity similar ambiguities produce total inconsistency of the theory beyond the one loop level, because at higher loops the emergence of the non-symmetric counterterms looks unavoidable. So, we can conclude that, in general, conformal invariant theories are not consistent at quantum level. In fact, the local conformal symmetry may be only approximate, despite it is a very useful tool for calculating quantum corrections.

\section{Acknowledgments.}

Author is very grateful to his collaborators on the subject, especially to M. Asorey, E. Gorbar, G. de Berredo-Peixoto and J. Solà for numerous discussions and common works. I am also thankful to the organizers of the Meeting for invitation to give this talk and for support. The work has been partially supported by the PRONEX project and research grants from FAPEMIG (MG, Brazil) and CNPq (Brazil) and by the fellowships from CNPq and ICTP (Italy). The text of this Proceedings has been completed during my visit to Theory Division of CERN and I would like to thank them for support and hospitality.

\section{References}

[1] V. Faraoni, E. Gunzig and P. Nardone, Conformal transformations in classical gravitational theories and in cosmology, Fund. Cosmic Phys. 20 (1999) 121 [gr-qc/9811047].

[2] J.C.Fabris, A.M.Pelinson and I.L.Shapiro, On the gravitational waves on the background of anomaly-induced inflation, Nucl. Phys. B597 (2001) 539 [hep-th/0009197].

[3] I.L. Shapiro and H. Takata, One-loop renormalization of the four-dimensional theory for quantum dilaton gravity, Phys. Rev. D52 (1995) 2162 [hep-th 9502111]; Conformal transformation in gravity, Phys. Lett. B361 (1995) 31 [hep-th/9504162]. 
[4] J. O'Hanlon, Intermediate-range gravity - a generally covariant model, Phys. Rev. Lett. 29 (1972) 137.

[5] S.L. Adler, Einstein gravity as a symmetry breaking effect in quantum field theory, Rev. Mod. Phys. 54 (1982) 729.

[6] I. L. Buchbinder, Mechanism For Induction Of Einstein Gravitation, Sov. J. Phys. 31 (1986) 77.

[7] I.L. Shapiro, Hilbert-Einstein Action from Induced Gravity coupled with Scalar Field, Mod. Phys. Lett. 9A (1994) 1985 [hep-th/9403077].

[8] I.L. Shapiro and G. Cognola, Interaction of Low - Energy Induced Gravity with Quantized Matter and Phase Transition Induced by Curvature, Phys. Rev. D51 (1995) 2775 [hep-th/9406027]; Back reaction of vacuum and the renormalization group flow from the conformal fixed point, Class. Quant. Grav. 15 (1998) 3411 [hep-th/9804119].

[9] S. Paneitz, A Quartic Conformally Covariant Differential Operator for Arbitrary Pseudo-Riemannian Manifolds, MIT preprint, 1983.

[10] R.J. Riegert, A Nonlocal Action For The Trace Anomaly. Phys. Lett. B134 (1984) 56;

E.S. Fradkin and A.A. Tseytlin, Conformal Anomaly In Weyl Theory And Anomaly Free Superconformal Theories, Phys. Lett. B134 (1984) 187.

[11] I. Antoniadis, J. Iliopoulos and T. N. Tomaras, On The Stability Of Background Solutions In Conformal Gravity, Nucl. Phys. B261 (1985) 157.

[12] J. A. de Barros and I.L. Shapiro, Renormalization group study of the higher derivative conformal scalar model, Phys. Lett. B412 (1997) 242 [hep-th/9706123].

[13] E. Elizalde, A.G. Zhecksenaev, S.D. Odintsov and I.L. Shapiro, One-loop renormalization and asymptotic behavior of a higher-derivative scalar theory in curved spacetime, Phys. Lett. B328 (1994) 297 [hep-th/9402154].

[14] E.S. Fradkin and A.A. Tseytlin, Conformal Supergravity, Phys. Repts. 119 (1985) 233.

[15] G. de Berredo-Peixoto and I.L. Shapiro, The higher derivative fermionic operator and trace anomaly, Phys. Lett. B514 (2001) 377 [hep-th/0101158].

[16] J. Erdmenger, Conformally covariant differential operators: Properties and applications, Class. Quant. Grav. 14 (1997) 2061 [hep-th/9704108]; Conformally covariant differential operators: Symmetric tensor fields. J. Erdmenger and H. Osborn, Class. Quant. Grav. 15 (1998) 273 [gr-qc/9708040].

[17] T.P. Branson, Conformaly covariant equations on differential forms, Comm. Part. Diff. Eq. 7 (1982) 393; An anomaly associated with 4-dimensional quantum gravity, Commun. Math. Phys. 178 (1996) 301.

[18] I.L. Buchbinder, S.D. Odintsov, I.L. Shapiro, Effective Action in Quantum Gravity, IOP Publishing, Bristol, 1992.

[19] I.L. Buchbinder, Renormalization-group equations in curved space-time, Theor. Math. Phys. 61 (1984) 393.

[20] S.W. Hawking, Zeta Function Regularization Of Path Integrals In Curved Space-Time, Comm. Math. Phys. 55 (1977) 133.

[21] N.D. Birrell, P.C.W. Davies, Quantum fields in curved space, Cambridge Univ. Press, Cambridge, 1982. 
[22] M.J. Duff, Twenty years of the Weyl anomaly, Class. Quant. Grav. 11 (1994) 1387 [hep-th/9308075].

[23] M. Asorey, E.V. Gorbar and I.L. Shapiro, Universality and Ambiguities of the Conformal Anomaly. Class. Quant. Grav. 21 (2003) 163 [hep-th/0307187].

[24] M.J. Duff, Observations On Conformal Anomalies, Nucl. Phys. B125 (1977) 334;

S. Deser, M.J. Duff and C. Isham, Nonlocal Conformal Anomalies, Nucl. Phys. B111 (1976) 45.

[25] S.M. Christensen, Vacuum Expectation Value Of The Stress Tensor In An Arbitrary Curved Background: The Covariant Point Separation Method, Phys. Rev. D14 (1976) 2490; Regularization, Renormalization, And Covariant Geodesic Point Separation, D17 (1978) 948.

[26] M. Asorey, G. de Berredo-Peixoto and I.L. Shapiro, Renormalization Ambiguities and Conformal Anomaly in Metric-Scalar Backgrounds, hep-th/0609138.

[27] S. Deser and A. Schwimmer, Geometric Classification of Conformal Anomalies in Arbitrary Dimensions, Phys. Lett. 309B (1993) 279 [hep-th/9302047]

S. Deser, Closed form effective conformal anomaly actions in $D \geq 4$, Phys. Lett. 479B (2000) 315 [hep-th/9911129].

[28] A.O. Barvinsky, Yu.V. Gusev, G.A. Vilkovisky and V.V. Zhitnikov, The One loop effective action and trace anomaly in four-dimensions, Nucl.Phys. B439 (1995) 561 [hep-th/9404187].

[29] D. F. Carneiro, E. A. Freitas, B. Gonçalves, A. G. de Lima and I. L. Shapiro, On Useful Conformal Tranformations In General Relativity, Grav. and Cosm. 40 (2004) 305 [gr-qc/0412113].

[30] I.L. Buchbinder, S.D. Odintsov and I.L. Shapiro, Nonsingular cosmological model with torsion induced by vacuum quantum effects, Phys. Lett. B162 (1985) 92;

J.A. Helayel-Neto, A. Penna-Firme and I. L. Shapiro, Conformal symmetry, anomaly and effective action for metric-scalar gravity with torsion, Phys. Lett. B479 (2000) 411 [gr-qc/9907081].

[31] I.L. Shapiro, J. Solà, Massive fields temper anomaly-induced inflation, Phys. Lett. B530 (2002) 10 [hep-ph/0104182];

A.M. Pelinson, I.L. Shapiro, F.I. Takakura, On the stability of the anomaly-induced inflation, Nucl. Phys. B648 (2003) 417 [hep-ph/0208184].

[32] I. L. Shapiro and A. G. Jacksenaev, Gauge dependence in higher derivative quantum gravity and the conformal anomaly problem, Phys. Lett. B324 (1994) 284.

[33] P. O. Mazur and E. Mottola, Weyl cohomology and the effective action for conformal anomalies, Phys. Rev. D64 (2001) 104022.

[34] R. Balbinot, A. Fabbri and I.L. Shapiro, Anomaly induced effective actions and Hawking radiation, Phys. Rev. Lett. 83 (1999) 1494 [hep-th/9904074]; Vacuum polarization in Schwarzschild space-time by anomaly induced effective actions and Hawking radiation, Nucl. Phys. B559 (1999) 301 [hep-th/9904162].

[35] E.V. Gorbar, I.L. Shapiro, Renormalization Group and Decoupling in Curved Space. JHEP 02 (2003) 021 [hep-ph/0210388]; Renormalization Group and Decoupling in Curved Space: II. The Standard Model and Beyond. JHEP 06 (2003) 004 [hep-ph/0303124].

[36] E.S. Fradkin and A.A. Tseytlin, Higher Derivative Quantum Gravity: One Loop Counterterms And Asymptotic Freedom, Nucl. Phys. B201 (1982) 469.

[37] I.L. Buchbinder and I.L. Shapiro, On the influence of the gravitational interaction on the behavior of the effective constants of Yukawa and scalar coupling, Sov. J. Nucl. Phys. 44 (1986) 1033. 
[38] K.S. Stelle, Renormalization Of Higher Derivative Quantum Gravity, Phys. Rev. D16 (1977) 953.

[39] B.L. Voronov and I.V. Tyutin, On renormalization of $R^{2}$ gravitation, Yad. Fiz. (Sov. Journ. Nucl. Phys.) 39 (1984) 998.

[40] I. Antoniadis, P.O. Mazur and E. Mottola, Conformal symmetry and central charges in four-dimensions, Nucl. Phys. B388 (1992) 627 [hep-th/9205015].

[41] G. de Berredo-Peixoto and I.L. Shapiro, Conformal Quantum Gravity with the Gauss-Bonnet term, Phys. Rev. D70 (2004) 044024 [hep-th/0307030].

[42] F. Englert, C. Truffin and R. Gastmans, Conformal Invariance In Quantum Gravity, Nucl.Phys. B117 (1976) 407;

E.S. Fradkin and G.A. Vilkovisky, Conformal Off Mass Shell Extension And Elimination Of Conformal Anomalies In Quantum Gravity, Phys. Lett. B73 (1978) 209. 